\title{
Two Point Fixation in ZMC Fracture-A Case Report
}

\author{
Akshay Kumar ${ }^{1}$ Abhigyan Manas ${ }^{2}$ Deepak Singh ${ }^{3}$ Shishir Dhar ${ }^{4}$ Amit Kumar Verma ${ }^{5}$ \\ Pramod Yadav 6 \\ 1 Oral and Maxillofacial Surgeon, CH BSF Srinagar, India. \\ 2,3Oral and Maxillofacial Surgeon, Career Post Graduate Institute of Dental Sciences and Hospital, \\ Lucknow, India. \\ ${ }^{4}$ Oral and Maxillofacial Surgeon, GMC, Saharanpur, India. \\ 5 Prosthodontist and Oral Implantologist, ESIC Hospital, Behrampur, Bengal, India. \\ ${ }^{6}$ Department of Periodontics and Community Dentistry, Dr. Z. A. Dental College AMU Aligarh, India.
}

\section{Case Report}

\section{Address for Correspondence Author}

Dr. Pramod Kumar Yadav, Department of Periodontics \& Community Dentistry, Dr. Z. A. Dental College, AMU, Aligarh, Uttar Pradesh, India.

E-mail: pramod468@gmail.com

Crossref doi: https://doi.org/10.36437/ijdrd.2020.2.2.0

\section{ABSTRACT}

Tripod fractures constitute of the zygomatic bone and its four articulations viz; frontal bone, maxillary bone, sphenoid bone, and temporal bone. Whenever a Tripod fracture happens, one or more of these articulations are interrupted. Personal altercations, Assaults, Road traffic accidents are the common causes leading to fracture of the zygomatic bone. The zygomaticomaxillary complex is one of the principle buttresses of the face and helps in transmitting the occlusal forces to the skull base. Anatomic disfigurement caused by zygomatic fracture warrants intervention tore establish facial symmetry. It is most predictably restored to premorbid condition by ORIF and AO (Arbeitsgemeinschaft für Osteosynthesefragen) miniplate osteosynthesis has proven to be an invaluable aid when used in severe craniomaxillofacial trauma.

Keywords: AO , Fracture, Fixation, Mimiplate.

Introduction: Fractures of the facial bones have been recognized for a long time, and attempt to treat such fractures has been recorded as far back as 25-30 centuries BC. Zygomatico-maxillary complex (ZMC) fractures are common after maxillofacial injuries. The various causes of fractures are trauma due to road traffic accidents, assaults, falls, sports-related injuries, and civilian warfare. (1) Up to $40 \%$ of facial fractures constitute Tripod fractures,(2)male population is more affected with an incidence of F: M- 1: 4(3). The zygomatic arch, a laterally positioned element of the craniofacial skeleton comprised of the zygoma and temporal bone, is susceptible to local trauma. Isolated zygomatic arch fractures comprise about $10 \%$ of all 
zygomatic fractures and result in noticeable depression at the arch fracture site. There may be impingement of the fractured arch on the coronoid process, resulting in limited mouth opening. (4)

Mini plates have revolutionized the standards of management of maxillofacial fractures by providing rigid internal fixation and ultimately better stability and less complications. Zygomatic bone is attached to adjacent bones and has four suture lines; which are the areas of anatomical weakness. Tripod fracture can be managed with one point or two point fixation; however, the numbers of sites of rigid fixation still remain a topic of debate. $(5,6)$

Case Report: A 33 Year old male reported to our hospital with history of road traffic accident two days back wiht reduced mouth opening, depressed zygomatic bone and circumorbital ecchymosis on right side. No history of loss of consciousness, nasal and ear bleed was present. Routine blood investigation and radiographs were advised to patient. Radiographic investigations revealed fractured right zygomaticomaxillary complex. Ophthalmic opinion was taken .Treatment was explained to the patient and informed written consent was obtained prior to surgery. (Figure.1)

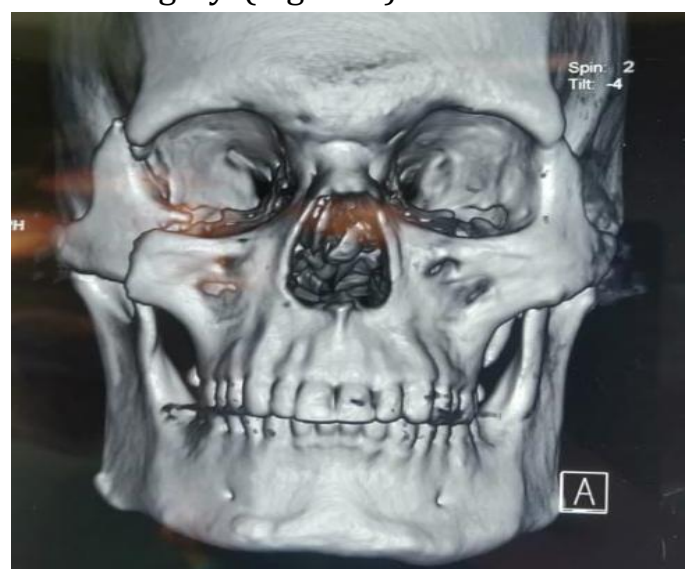

Figure1: CT Showing Right ZMC Fracture

Local anaesthesia with adrenaline 1:80,000 was infiltrated and lateral eyebrow incision approximately $1.5 \mathrm{~cm}$ in length was placed over right fronto-zygomatic suture region (fig 2). Skin over the supra orbital rim region should be supported using two fingers. Periosteal elevator should be used while drilling to protect the eyeball from any injury. First hole should always be drilled in the unstable segment of the fracture. AO titanium locking plate was adapted and fixation was done with the help of $6 \mathrm{~mm}$ screws. (figure.2)

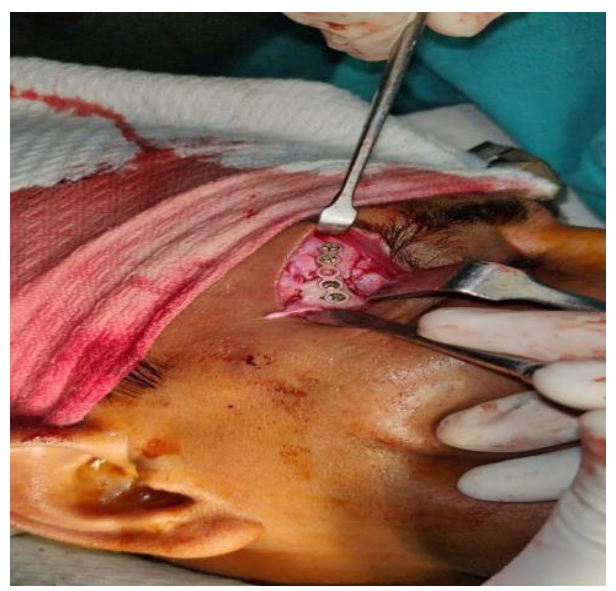

Figure 2: AO Miniplate Fixation at Right Frontozygomatic Region 
Transoral (Keen) lateral maxillary vestibular incision approximately $2 \mathrm{~cm}$ was made with a scalpal. Fracture site was exposed, elevated and reduced to its anatomical position. Right AO titanium locking LPlate was adapted and finally all screws were tightened. (figure.3)

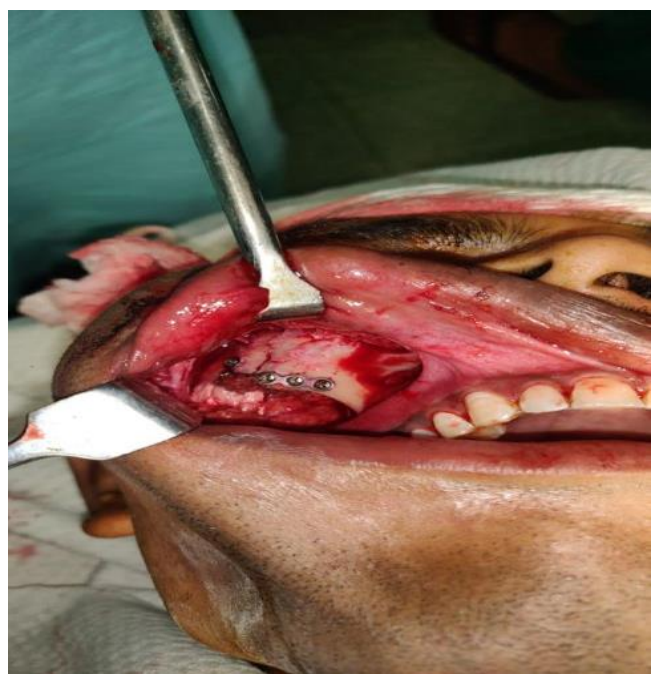

Figure 3: AO Miniplate Fixation at Right Zygomatic Buttress Region

Wound was closed in layers using 3-0 vicryl and 5-0 proline sutures. Post operatively antibiotics and analgesic are prescribed. Suture removal was done on $10^{\text {th }}$ postoperative day. Healing was uneventful. Post operative radiograph was taken after surgery. (figure. 4)

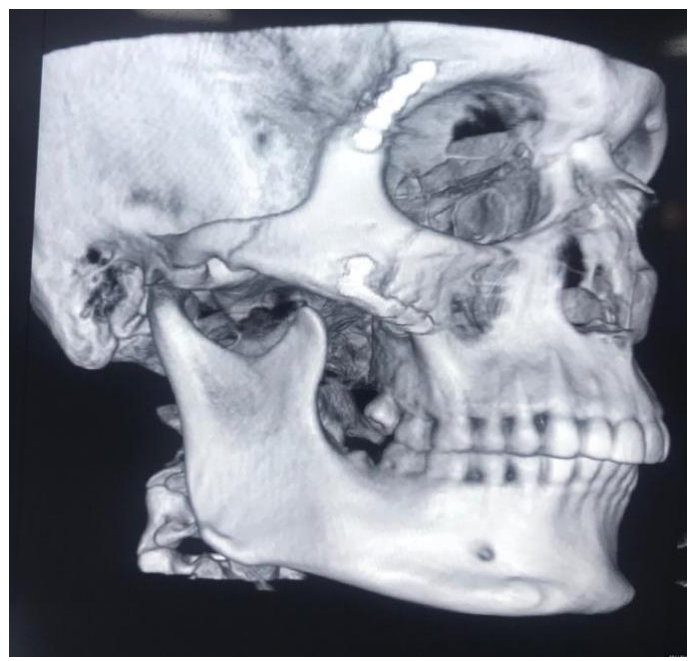

Figure 4: Post operative CT scan showing reduction and stabilization Patient was called on for periodic follow up and Patient had an almost imperceptible scar.

Discussion: Two-point fixation has been quoted by many authors in the English literature, but very few have applied it clinically and have highlighted its advantages. Two-point fixation has been used sporadically by few surgeons predominantly for fixation of the ZMC region at fronto-zygomatic suture and zygomatic buttress. The integrity of the zygomatic complex is critical in maintaining normal facial width and cheek prominence. Zygomatic bone alone rarely gets fractured; its articulating surfaces: 
maxilla, temporal, frontal and sphenoid are also involved. According to a study done by Ellis and Kittidumkerng who studied a variety of surgical approaches, the maxillary vestibular approach was used more frequently, either alone or in combination with other approaches. The next frequently used approach was through the lower eyelid. Complications associated with maxillary vestibular approach were not significant, and approximately $20 \%$ of those having lower eyelid approach had some amount of scleral show. (7)

Peter B Grey et al (8) advocated ophthalmic opinion is mandatory in most of the zygomatic complex fractures; as delayed retrobulbar hemorrhage may lead to blindness. Keen lateral vestibular and zygomatico frontal suture approach was used to treat the displaced zygomatic complex fractures. (9)Six $\mathrm{mm}$ screws can be safely placed above the frontozygomatic suture. Infra orbital rim incision provided adequate exposure of the fracture site; fractured bony segments were accurately aligned and fixed using AO locking miniplate.

The results of study conducted by O'Hara et al. confirmed that the best site for rigid fixation is the zygomatico-maxillary buttress since this is the direct antagonist to pull of the masseter muscle along with the fact that fixation placed was deep and plates were rarely felt in that area, and therefore, a longer and stronger fixation plate could always be used in this site. The infraorbital rim though one of the points of fracture that requires reduction is the least important site for fracture fixation. Similarly, fronto-zygomatic suture line represents very thick bone which was ideal for rigid fixation. ${ }^{(10)}$ Hence, we conducted a report on two-point fixation to further evaluate the treatment outcome in terms of esthetics, function, stability, and incidence of complication.

Conclusion: In this zygomatico-maxillarycomplex fracture treated with two-point fixation the results were satisfactory in terms of both esthetics and function with elimination of external scar at infraorbital rim. Severely displaced zygomatic complex fractures may require additional points of fixation.

\section{References:}

1. Gomes PP, Passeri LA, Barbosa JR. A 5-year retrospective study of zygomatico-orbital complex and zygomatic arch fractures in Sao Paulo state, Brazil. J Oral Maxillofac Surg 2006; 64:63-7.

2. Kim JH, Lee JH, Hong SM, Park $\mathrm{CH}$.The Effectiveness of 1-Point Fixation for Zygomaticomaxillary Complex Fractures: ArchOtolaryngol Head Neck Surg. 2012;138(9):828832.

3. Vatsa R, Priyadarshini P, Singh A.D.B. Comparison of One Point Fixation Versus Two Point Fixation in the Treatment of Zygomatico Maxillary Complex Fractures. Int Health c Res J 2018; 2(9):229-234. doi: https://doi.org/10.26440/ihrj.v2i9.177

4. Yamamoto K, Murakami K, Sugiura T, Fujimoto M, Inoue M, Kawakami M, et al. Clinical analysis of isolated zygomatic arch fractures. J Oral Maxillofac Surg 2007;65:457-61.

5. Parashar A, Sharma R K, Makkar S .Rigid internal fixation of zygoma fractures: A comparison of two-point and three-point fixation :Indian J PlastSurg January-June 2007 Vol 40 Issue 1

6. JiSY, KimSS, Kim MH, Yang WS. Surgical Methods of Zygomaticomaxillary Complex Fracture: Arch CraniofacSurg Vol.17 No.4, 206-210. 
7. Ellis E $3^{\text {rd }}$, Kittidumkerng W. Analysis of treatment for isolated zygomaticomaxillary complex fractures. J Oral Maxillofac Surg 1996;54:386-400.

8. Gray B.P, Leen MM and Loftus M. J. Late retrobulbar hemorrhage and blindness following malar fracture complicated by factor XI deficiency. Journal of Oral and Maxillofacial Surgery 1993; 51: 699-702.

9. Olate, Monteiro L, Renato S,Roger W F, Marcio M. Surgical Approaches and Fixation Patterns in Zygomatic Complex Fractures. Journal of Craniofacial Surgery2010; 21(4): 1213-1217.

10. O'Hara DE, DelVecchio DA, Bartlett SP, Whitaker LA. The role of microfixation in malar fractures: A quantitative biophysical study. Plast Reconstr Surg 1996; 97:345-50.

How to cite this Article: Akshay Kumar ${ }^{1}$ Abhigyan Manas ${ }^{2}$ Deepak Singh ${ }^{3}$ Shishir Dhar ${ }^{4}$ Amit Kumar Verma ${ }^{5}$ Pramod Yadav6; Two Point Fixation in ZMC Fracture - A Case Report

Int. J. Drug Res. Dental Sci., 2020; 2(2): 3-7.

Source of Support: Nil, Conflict of Interest: None declared.

Received: 24-3-2020 Revised: 15-4-2020 Accepted: 18-4-2020 